\title{
Identification and Characterization of Trichoderma citrinoviride Isolated from Mushroom Fly-Infested Oak Log Beds Used for Shiitake Cultivation
}

\author{
Jun Young Kim ${ }^{1}$, Hyuk Woo Kwon ${ }^{1}$, Longqing Tang ${ }^{1}$ and Seong Hwan Kim ${ }^{1,2 *}$ \\ ${ }^{1}$ Department of Microbiology and ${ }^{2}$ Institute of Basic Sciences, Dankook University, Cheonan 330-714, Korea \\ (Received on March 21, 2011; Revised on June 16, 2011; Accepted on June 16, 2011)
}

Recently, mushroom flies have been infesting in oak log beds used for shiitake cultivation in many mushroom farms all over Korea (Shin et al., 2011). Some shiitake farmers argue that the fly-infested oak log beds do not produce or reduce the fruiting body of shiitake, resulting in huge economic loss. Other shiitake farmers assume that the mushroom fly vectors green mold disease in infested oak log beds. In an effort to chase the green mold disease, we sampled mushroom fly-infested shiitake log beds from a mushroom farm located in Cheonan, Korea in June, 2009. When we debarked the sampled shiitake log bed, the mushroom fly's larvae were present in the brown colored log bed (Fig. 1A, B). The fungus DUCC001 was isolated from the brown colored log bed and deposited at Dankook University Culture Collection (DUCC).

The fungus DUCC001 produced pale yellow pigment on PDA and formed numerous conidia around inoculums on Czapek yeast extract agar (CYA) (Fig. 1C, D). Optimal growth temperature and $\mathrm{pH}$ for the fungus were $30^{\circ} \mathrm{C}$ and $\mathrm{pH} 5$, respectively. Typical morphological features of Trichoderma were observed in the fungus DUCC001 by a phase contrast Axioskop 40 (Karl Ziess, Germany) and a scanning electron microscope (Hitachi S-4200). Conidiophores were hyaline, $5.0-3.0 \mu \mathrm{m} \times 3.0-1.5 \mu \mathrm{m}$ and branched in a pyramidal fashion shape. Phialides were flask-shaped (Fig. 1E). Conidial mass was green. Conidia size was $2.3-2.8 \mu \mathrm{m} \times 1.5-2.0 \mu \mathrm{m}$ and elliptic shape(Fig. 1F). Unicellular subglobose chlamydospore-like structure was also observed (Fig. 1G).

Trichoderma is one of complex fungal groups that are difficult to identify solely based on morphological characters. Thus, to confirm the identity of the fungus DUCC001 at the molecular level, partial translation elongation factor $1 \alpha(t e f-1 \alpha)$ gene was amplified by PCR with TEF728 and TEF1 primers (Evidente et al., 2008), cloned and sequenced. The resulting sequence of $651 \mathrm{bp}$ was deposited in GenBank(JF700485). The sequence of the fungus DUCC001 shared $99 \%$ sequence identity with that of T. citrinoviride (EU338334) in GenBank and by Tricho-BLAST at the website of the International Subcommission on Trichoderma and Hypocrea Taxonomy (ISTH, $\mathrm{http}: / /$ www.isth.info/). A phylogram based on tef-1 $\alpha$ gene sequences revealed that the fungus DUCC001 was grouped with $T$. citrinoviride (Fig. 2).

To check the invading ability of the $T$. citrinoviride DUCC001 in a shiitake log bed, we inoculated the fungus on a Mongolian oak log bed ( $15 \mathrm{~cm}$ in diameter) that was precolonized by the mycelia of a shiitake strain and incubated in a mushroom cultivation house (at

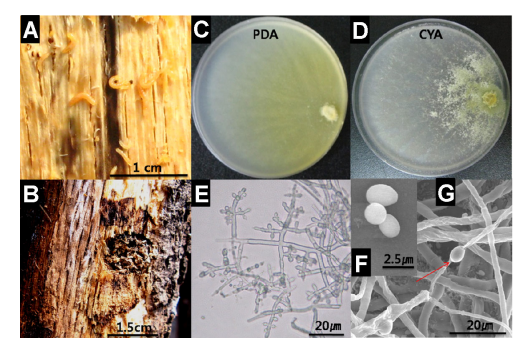

Fig. 1. A, B: Damaged shiitake log beds infested by mushroom fly's larvae. Fig. 1. A, B: Damaged shiitake log beds infested by mushroom fly's larvae.
C, D: Colony characteristics of the fungus DUCC001 grown on PDA and CYA at 25 for 7 days. E, F: Light microscopic image of conidiophores and conidia of the fungus DUCC001. G: SEM micrograph of a chlamydosporelike structure of the fungus DUCC 001 .

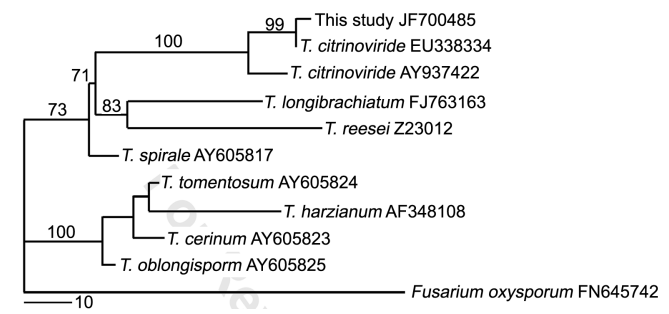

Fig. 2. Phylogenetic relationships of the fungus DUCC001 to other related species. Phylogram was constructed based on tef- $1 \alpha$ gene sequences by the neighbor-joining method in PAUP* program. Numbers above branches represent bootstrap values with 1,000 replications.

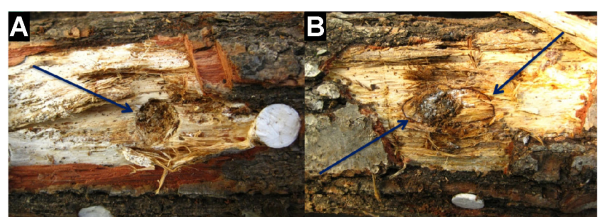

Fig. 3. Artificial inoculation of Trichoderma citrinoviride DUCC001 on oak log beds precolonized with the mycelia of a shiitake strain. After debarking, the surface features of oak log beds were shown. A: Sterilized sawdust media without T. citrinoviride DUCC001 (control). The arrow indicates the presence of white healthy shiitake mycelia near the inoculated hole. B: Inoculated using T. citrinoviride DUCC001-grown sawdust media. The arrows indicate the formation of browning zone near the inoculated hole, which is typical sign formation of browning zone near the inoculated hole, which is typical sign
found on the debarked surface of mushroom-fly damaged shiitake log beds.

avg. temp $26^{\circ} \mathrm{C}$, relative humidity $85 \%$ ) for 54 days. After taking out the bark near the inoculation hole, we found a brown colored zone that clearly formed around the inoculation hole. The brown zone was not found in the control inoculation. From the brown colored zone we could isolate again the inoculated $T$. citrinoviride DUCC001. This result indicates that $T$. citrinoviride is able to colonize the shiitake log bed. To our knowledge, this is first report of T. citrinoviride damage in oak $\log$ beds used for shiitake cultivation. So far, T. citrinoviride disease has been reported from oyster and button mushroom in Korea (Park et al., 2005).

\section{Acknowledgement}

This study was carried out with the support of Forest Science \& Technology Projects (Project No. S120909L050000) provided by the Korea Forest Service and Technology Development Program for Agriculture and Forestry, Ministry for Food, Agriculture, Forestry and Fisheries, Republic of Korea.

\section{References}

Evidente,A., Ricciardiello, G.,Andolfi,A., Sabatini, M. A., Ganassi, S., Altomare, C., Favilla, M. and Melck, D. 2008. Citrantifidiene and citrantifidiol: bioactive metabolites produced by Trichoderma citrinoviride with potential anitifeedant activity toward aphids.J. Agric. Food. Chem. 56:3569-3573.

Park, M. S., Seo, G. S., Lee, K. H., Bae, K. S. and Yu, S. H. 2005. Morphological and cultural characteristics of Trichoderma spp. associated with green mold of oyster mushroom in Korea. Plant Pathol. J. 21:221-228.

Shin, S., Lee, H. and Lee, S. 2011. Two cecidomyiid gall midge (Diptera: Cecidomyiidae) pests of shiitake mushrooms (Agaricales: Marasmiaceae). J. Asia Pac. Entomol. 14:387-391.

*Corresponding author (piceae@dankook.ac.kr) 